\title{
Biological, ethological, genetical, environmental, clinical and pharmacological alliance towards obesity control: the target of "The obese species workshop" in Erice, 21-26 October 2011
}

\author{
Samir G. Sukkar • Carlo Polidori
}

Published online: 24 November 2011

(C) Springer-Verlag 2011

The Obese Species congress, featuring a multidisciplinary approach to obesity, was held in Erice October 21-26, 2011, organized by the International School of Ethology of the Majorana Foundation. The introductory lecture by John Blundell provided an overview of the development of the research field of obesity, going from the model of energy expenditure with homeostatic control of appetite to environmental interaction defining susceptible and resistant human phenotypes. He presented an example of phenotypes, noting that while $80 \%$ of the obese population loses body weight under physical exercise, as would be expected, a full $20 \%$ gains weight. A genetic approach to brain control of appetite was described by Bradford Lowell. Using Cre/Lox technology, he showed the importance of the antiobesity effect of the leptin-gabaergic system, the Agouti related peptide and glutamatergic brain system in food-seeking activity. The brain and gut communication has been the target of study for treatment of obesity for several years with little success. Matthias Tschop showed that concomitant injection of gut peptides such as GLP1, GIP and glucagone can decrease food intake in humans, suggesting that polydrug therapy should be the basis for treating an obese person.

The first day concluded with Daniele Piomelli speaking of the "cannabinoid fiasco" of rimonabant in the control of

\section{S. G. Sukkar $(\bowtie)$}

Dir. Resp.le U.O.D. Dietetica e Nutrizione Clinica,

Az. Ospedale-Università San Martino di Genova,

Università di Genova, Pad. VII piano $2^{\circ}$,

Largo R. Benzi 10, 16132 Genoa, Italy

e-mail:samir.sukkar@hsanmartino.it

C. Polidori

Università degli Studi di Camerino, Piazza Cavour 19/f,

62032 Camerino, MC, Italy human obesity; he indicated that the naturally occurring amide of ethanolamide and oleic acid could reduce feeding through a brain oxitocinergic mechanism.

Saverio Cinti discussed the role of brown and white fat tissue in the control of energy intake. Two considerations emerged. First, brown is a good fat and low temperature can increase its mass. Second, there is evidence that obesity is the consequence of adipose tissue inflammation.

Brown and white adipocytes are in equilibrium depending by environmental factor in particular food and temperature. The temperature can increase the BAT mass by a transdifferentiation process.

On the other hand, the necessity to energy storage following to excessive energy intake moves the BAT towards WAT. Moreover when WAT is too much due to its hypertrophy up to the critical death size inflammation could arise. Visceral adipocytes have a critical death size smaller than subcutaneous adipocytes, thus explaining the higher inflammation and higher morbidity of visceral fat. According to the hypothesis of Cinti, the smaller critical death size of visceral adipocytes is due to their main origin from brown adipocytes transformed into small white adipocytes less expansible that white ones.

Antonio Vidal-Puig described the Insig1/SREBP1/ SCD1 network, an allostatic regulatory loop designed to preserve white adipose tissue lipid omeostasis. Down regulation of this system leads to obesity-induced insulin resistance. The importance of early stress events in the control of depressive-like disorders as a contribution to eating disorders was discussed by Anna Moles. The role of stress in obesity was also pointed out by Zofia Zukowska and Alessandro Bartolomucci, who gave us a behavioral model to study new drugs that can interfere with hypothalamus-pituitary-adrenal axis function. The role of membrane lipids as components of cell membrane, serving 
in energy storage and as intermediates in signalling pathways was shown by Matej Oresic who discussed about lipidomic. These data confirm the Cinti hypothesis. As a matter of facts from the lipidome analysis of adipocytes membranes of fat versus lean subjects it has been, paradoxically observed that fat adipocyte membranes contain more polyunsatured.

The increase of polyunsaturated fatty acids promotes membrane fluidity so to raise adipocytes volume until the set point when they go through apoptosis becoming crowncells. In addition, the increase of polyunsaturated fatty acids into cell membranes, leads to a greater availability of inflammatory mediators that, together with the inflammatory chronic condition, makes easier the development of the metabolic syndrome.

Strong change in the composition of such lipids could lead to a new allostatic set point with activation of alternative biosynthetic pathways that in turn could lead to accumulation of unnecessary lipids. John Wilding indicated that subjects who achieve only limited weight loss through dieting should undergo pharmacological treatment to lose more. He explained the pros and cons of the many therapies available over the years. John Rodgers clarified that the adverse reaction due to the antiobesity drugs could have been prevented if a detailed behavioral satiety sequence, called feeding-grooming-resting, had been studied. A take home message from his speech is the value of polytherapy, though not all formulations work.

Jerrold Heindel underlined the importance of the environmental components on the development of obesity; there are a lot of endocrine disrupting chemicals (EDC) that specifically affect weight gain (this subclass is called obesogens). Data in humans support the view that exposure to EDCs during development can affect weight gain in infants and in children. The alteration or deregulation of the "endocrine set point" predispose individuals for developing obesity later in life.

The obesogen hypothesis shifts the focus of the disease etiology from classical genetics to a series of complex interactions that include epigenetic alterations combined with nutritional and environmental chemical exposures. This hypothesis changes the focus from intervention to prevention.

Among the EDCs, one is of great importance on the development of obesity and metabolic syndrome: the bisphenol A (BPA). Vom Saal focused on the effects of exposure to BPA. This chemical with estrogenic activity is one of the highest volume chemicals produced in the world. Data on rats show that gestational and neonatal exposure to BPA can increase postnatal growth rate and alter abdominal fat cell number and size, associated with changes in fat cell gene activity. In addition, the effect of BPA on metabolic processes depend on an interaction with components of the diet.

Fredrik Bäckhed said that it also seems that gut microbiota may regulate adiposity. Indeed, it has been observed that the microbiota in lean humans is more varied than those in obese people. Toxicology studies have also demonstrated that bisphenol A can disrupt the proper growth of adipose tissue. A striking association was noted in the prevalence of obesity in the population around factories that produce such compounds in the U.S. A.. The closing session of the congress addressed the obesity epidemic from an anthropological point of view. Jonathan Wells' evolutionary perspective brought a refreshingly original outlook to the subject. Over the ages, adiposity has been anything but deleterious for human evolution in response to environmental demands, but today perhaps the best strategy for preventing obesity lies in countering the manipulative economic forces that exploit our phenotypic flexibility. Last but not least was John Speakman's evolutionary overview of this epidemic disease. The 'thrifty' gene theory holds that alleles that promote metabolic efficiency were beneficial in uncertain environments at risk for famine, but now they contribute to widespread obesity. Inconsistencies in this theory have lead to proposal of a 'drifty' gene theory. With the advent of agriculture, fire exploitation, and weapons development, there was a 'drift' in the alleles of genes of weight regulation beneficial for famine survival and predation avoidance. In Speakman's words, today 'some people have been lucky in the genetic drift lottery and retained good control over their body weight in spite of the readily available food resources, while others have fared less well and become obese.' He challenged the tendency to attribute today's obesity etiology to environmental factors alone, spoke of assortative mating for obesity, and offered data to cast light on these hypotheses.

Contributors to the closing discussion pointed out that obesity has increased remarkably in the last half century and that genetic and environmental factors have been keys in this phenomenon. They indicated that some simple modifications should be made: daily exercise and lower fat intake top the list, while pharmaceutical intervention comes later, possibly with polytherapy, but emphasized that prevention is the most important tool at our disposal.

\section{S. G. Sukkar \\ Editor-in-Chief}

\section{Carlo Polidori}

